\title{
Primary leiomyosarcomata of the lung
}

\author{
$M . K . M A S O N^{1}$ AND $P$. S. AZEE $M^{2}$ \\ From the Department of Morbid Anatomy, King's College Hospital Medical School, and the Group \\ Laboratory, Lambeth Hospital, London
}

Primary sarcomata are uncommon in the lung, and few cases have been reported. The majority of these tumours have proved to be leiomyosarcomata; other varieties have been found very rarely. The literature on pulmonary leiomyosarcomata has been reviewed both by Agnos and Starkey (1958), who were able to find 18 previously recorded cases and added two of their own, and also by Yacoubian, Connolly, and Wylie (1958), who reported an additional case. There have since been accounts by Merritt and Parker (1957), who found the tumour in a child, and by Glennie, Harvey, and Jewsbury (1959), who described two further cases.

The diagnosis of any of these tumours is histological and is straightforward for welldifferentiated lesions. On the other hand, it may be difficult to distinguish between a poorly differentiated sarcoma and the common anaplastic carcinoma. It is possible that leiomyosarcoma may occur less infrequently than is generally believed. The following five cases are presented to show their main pathological and clinical features.

\section{CASE HISTORIES}

CASE 1 A man aged 75 was admitted with severe dyspnoea and cough of seven weeks' duration. Examination showed him to be cyanosed with signs of an effusion at the right base. He died three days after admission. Necropsy showed a firm, whitish, rounded tumour $6 \mathrm{~cm}$. in diameter in relation to the right main bronchus and obliterating the upper lobe bronchus. A metastasis was present in the liver.

Section of the tumour showed a well-differentiated fibroleiomyosarcoma with frequent muscle fibres forming bundles presenting a whorled appearance. The cells were elongated and the nuclei were ovoid. There was abundant cytoplasm. Many of these cells stained bright yellow by a polychrome method (Herovici, 1963) which stains muscle cells this colour. Van Gieson and trichrome stains showed bands of collagen lying between and close to the tumour cells.

1 Present address: Department of Pathology, St. James's Hospital, Leeds

2 Present address: Department of Pathology, Royal Free Hospital, London
About one mitosis was present per 10 high-power fields.

CASE 2 A 60-year-old man complained of severe dyspnoea and purulent sputum which followed an attack of bronchitis six months previously. He had had a cough for many years. Marked cyanosis of the face and extremities was noted on examination. Radiographs showed a circumscribed rounded lesion in the apex of the left lower lobe. A left lower lobe lobectomy was successfully performed, but the patient died of pulmonary embolus 13 days after operation. Examination of the specimen (Fig. 1) revealed a rounded, whitish-yellow, apparently encapsulated tumour peripherally situated in the apex of the left lower lobe.

Histologically, this was a smooth muscle tumour, similar to that found in case 1 (Fig. 2). Mitoses were

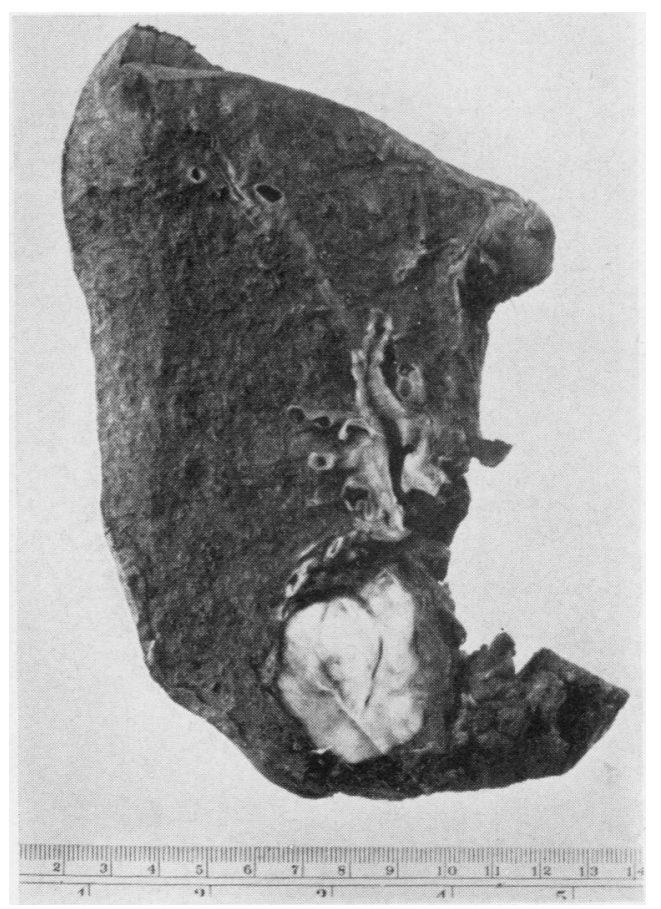

FIG. 1. Case 2. Macroscopic appearance of the tumour. 


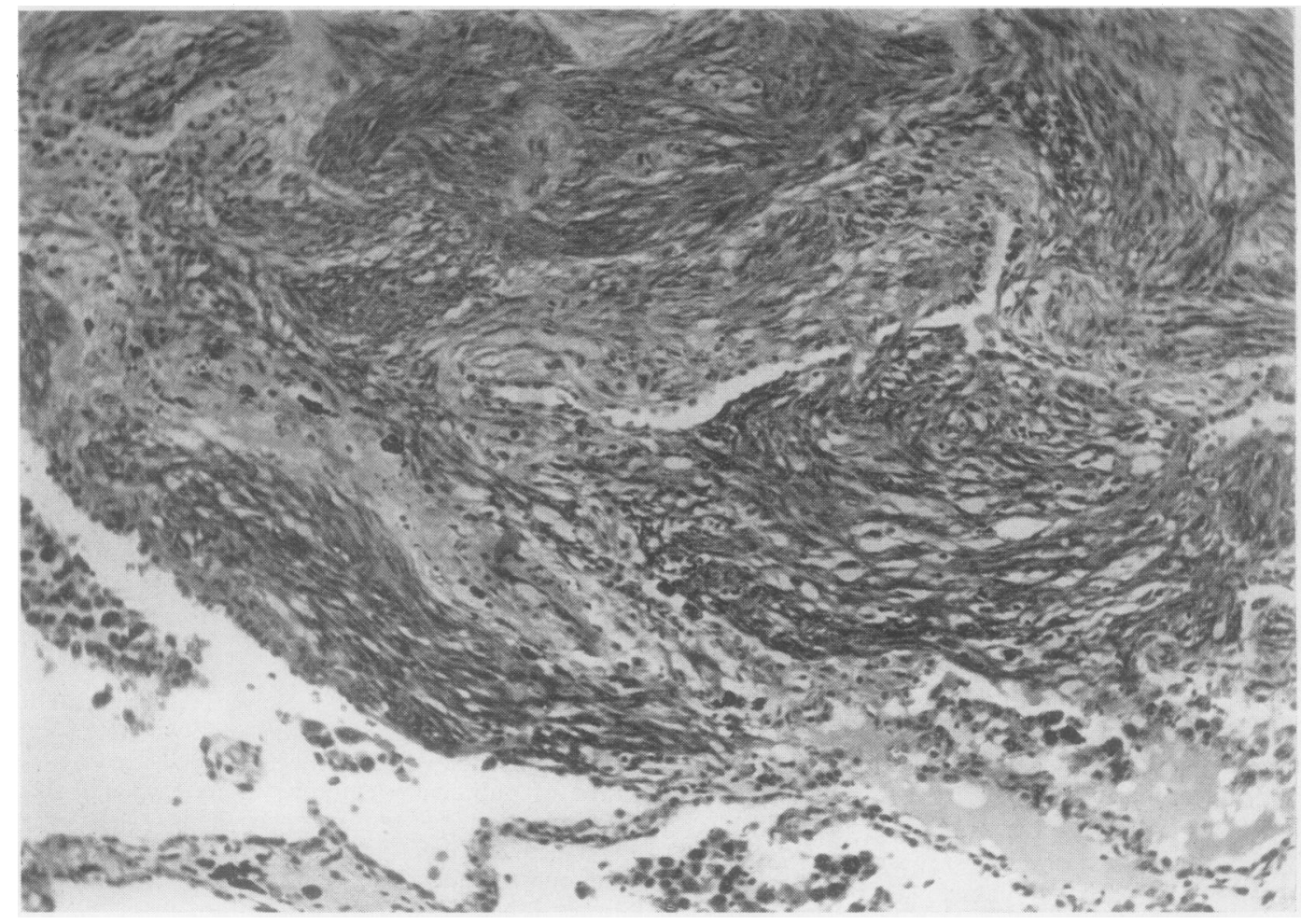

FIG. 2. Case 2. Section of the tumour showing a well-differentiated fibroleiomyosarcoma $(\times 150)$.

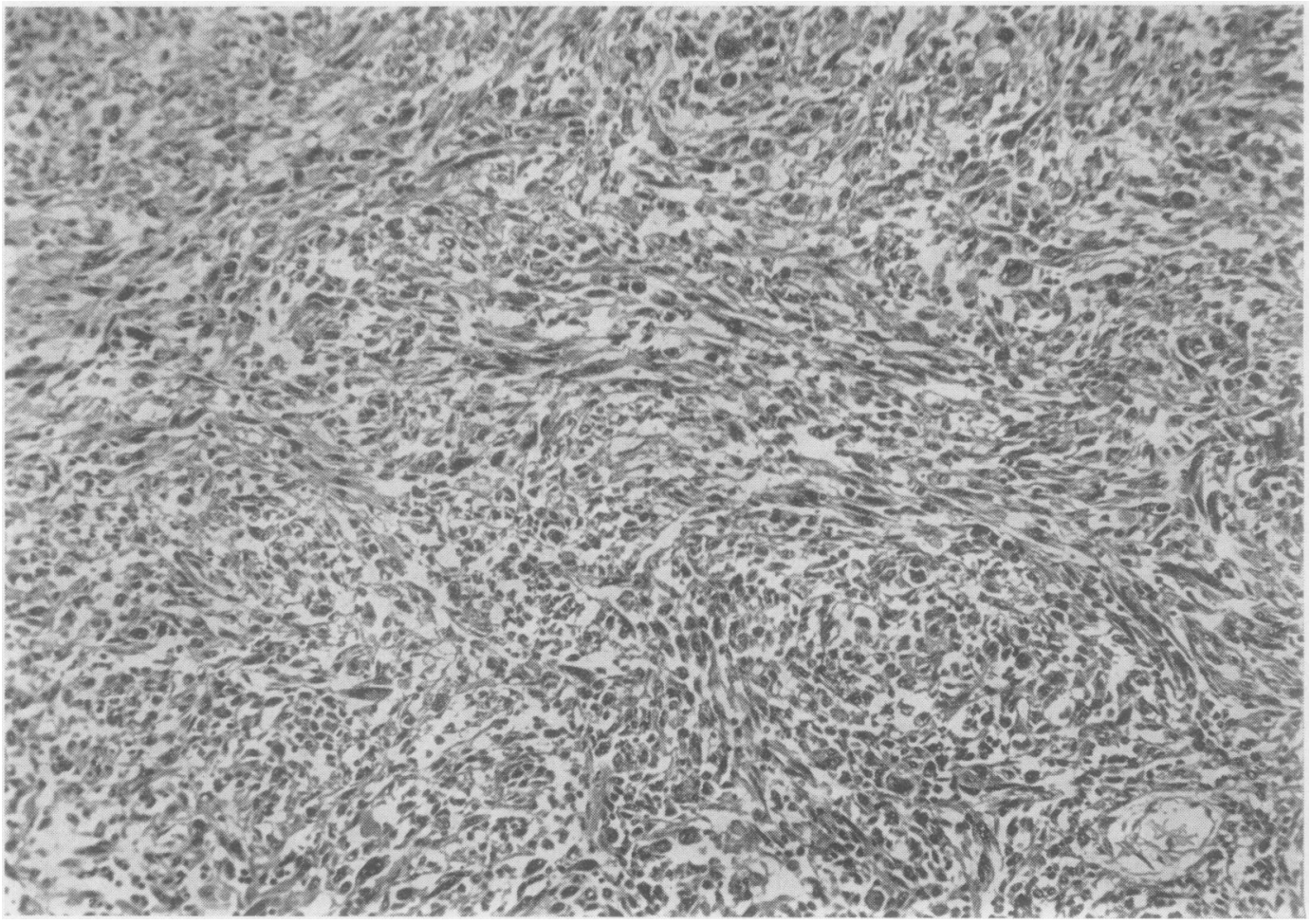

FIG. 3. Case 3. The tumour is less well differentiated than that shown in Fig. 2, but there is still an obvious whorled pattern $(\times 150)$. 
infrequent, and, although this was probably a welldifferentiated sarcoma, it was difficult to be certain that the lesion was not benign.

CASE 3 A man aged 70 had a 'stroke' one year before admission followed by Jacksonian fits. He gave no history of chest trouble. Examination showed a left hemiplegia, generalized rhonchi, and signs of consolidation at the right base. He died five days after admission. Necropsy revealed a firm, poorly circumscribed, greyish white tumour, $3 \mathrm{~cm}$. in diameter, in the right upper lobe and extending to the right lower lobe. A cerebral metastasis, $3 \mathrm{~cm}$. in diameter, was present in the right parietal region and a similar but smaller secondary in the left parietal region.

Section showed a less well differentiated tumour than was found in the two previous cases but a whorled pattern was present (Fig. 3). Mitoses were more frequent, about one in every two high-power fields. A considerable number of collagen fibres were seen close to the cells.

CASE 4 A 62-year-old man had a left upper lobectomy at another hospital for a malignant tumour. At that time, chest symptoms had been present for four months. He died nine months after this operation, and at necropsy an extensive recurrence of firm white tumour was found in the remaining lobe of the left lung with infiltration of the pericardium and pleura. Metastases were present in the hilar and mediastinal lymph nodes, liver, and adrenals. The histological features are described below with those of case 5 .

CASE 5 A man aged 59 presented with haemoptysis and chest pain of five weeks' duration. Cough and dyspnoea, recently much worse, had been present for many years. Chest radiographs showed a spherical mass in the left upper lobe. He died shortly after admission. Necropsy revealed emphysema and a rounded encapsulated tumour, $7 \mathrm{~cm}$. in diameter, with a necrotic centre, in the left upper lobe. No metastases were found.

Sections from cases 4 and 5 showed a similar histological appearance. Both tumours were poorly differentiated but presented the pattern of a fibroleiomyosarcoma, with bundles of cells in some areas giving a faint whorled pattern. The majority of cells were oval or spindle-shaped, but many bizarre and multinucleated cells were seen. Some of them stained yellow by the polychrome method. Approximately one mitosis per three high-power fields was present, and van Gieson and trichrome stains demonstrated collagen lying in relation to the tumour cells (Fig. 4).

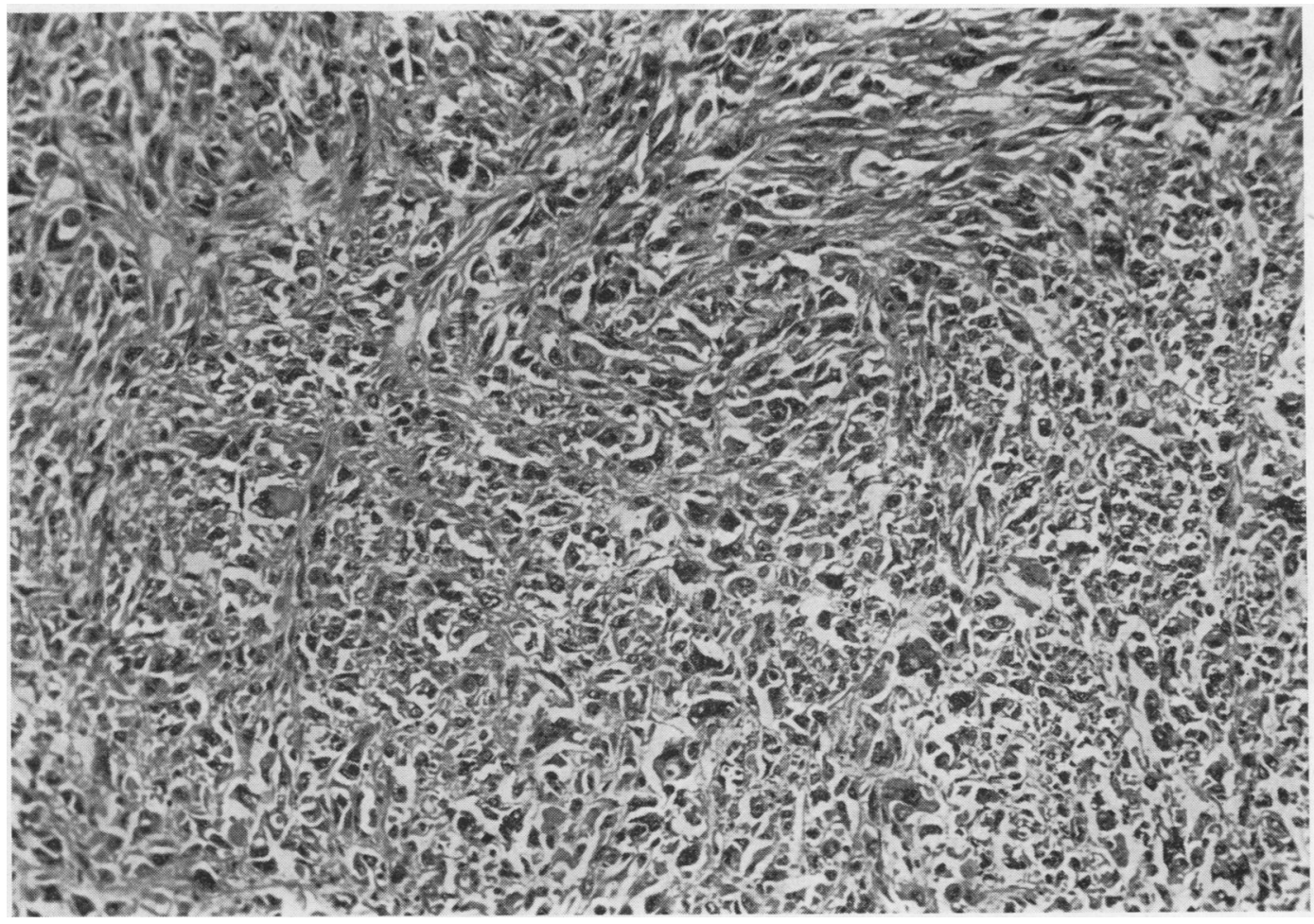

FIG. 4. Case 5. A poorly differentiated sarcoma. Many bizarre cells are present, but there is a suggestion of a whorled pattern $(\times 150)$. 


\section{DISCUSSION}

Fibroleiomyosarcomata may arise anywhere in the lung: sometimes they are in the periphery, sometimes they involve almost an entire lobe, and at other times they arise from a bronchus. They resemble carcinoma in their macroscopic appearances, except that they have a more fleshy appearance, and even the poorly differentiated tumours tend to be circumscribed and apparently encapsulated. Occasionally a whorled pattern may be seen with well-differentiated lesions. In the less common intrabronchial tumours, there may be a polypoid mass of growth extending up the lumen of the bronchus (Yacoubian et al., 1958'. Metastases are more frequent from the poorly differentiated tumours, and when present they are blood-borne. Although Yacoubian et al. regard the absence of lymph node metastases as one of the characteristics of these neoplasms, regional lymph node involvement was found in one case in this series.

Microscopically, the tumours range from welldifferentiated fibroleiomyosarcomata with easily demonstrable muscle cells to anaplastic tumours, $\overrightarrow{\vec{F}}$ in which the histological diagnosis is difficult and can only be made by careful study. Benign leiomyomata have been recorded in the lung in a small number of cases (Agnos and Starkey, 1958) and it may not be easy to distinguish between a benign tumour of this nature and one of low-grade malignancy. On rare occasions, such $\vec{\circ}$ a benign tumour may undergo malignant change (Glennie et al., 1959).

We consider that the poorly differentiated tumours in this series are sarcomata, most probably fibroleiomyosarcomata, and that the ? alternative diagnosis of anaplastic carcinoma is excluded for the following reasons: (1) macro- $\vec{\omega}$ scopic and microscopic evidence of encapsula- 을 tion ; (2) the presence of cells that are stained in $\rightarrow$ a similar manner to muscle by the polychrome technique; (3) collagen present in intimate relation to individual tumour cells, or sometimes $\stackrel{\rho}{2}$ small groups of cells, implying that they are laying it down ; (4) a tendency for the tumour to spread in and involve the interalveolar septa, which appear thickened, rather than to grow within the

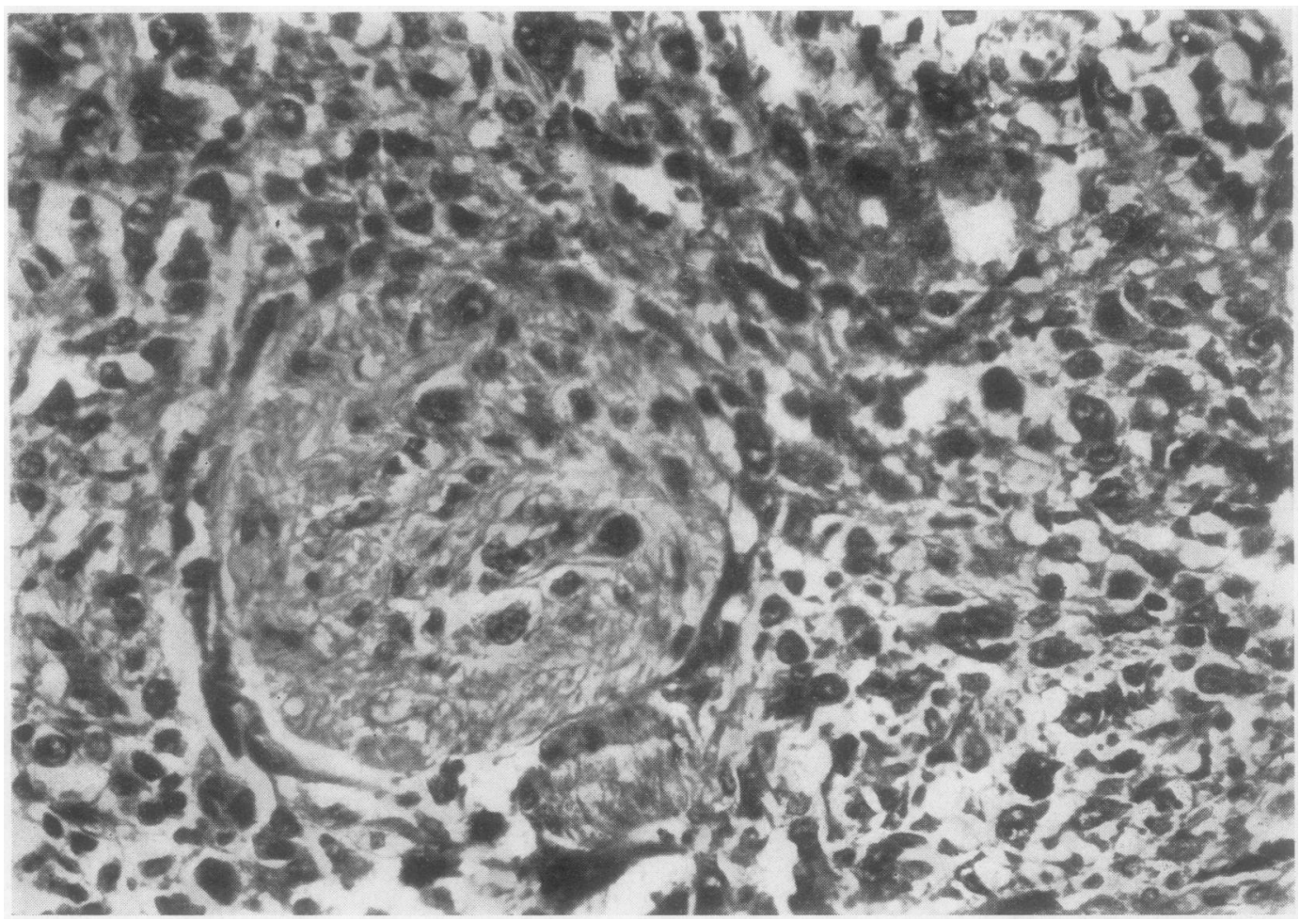

FIG. 5. Section of a tumour of neurogenic origin, showing malignant cells apparently arising from the nerve sheath $\stackrel{\unrhd}{\sigma}$ seen on the left $(\times 475)$. 
alveoli as is seen with carcinoma. The possibility that any of these tumours were metastases from a primary sarcoma of the uterus (Pierce, Alznauer, and Rolle, 1954) does not arise in this series as the patients were all men.

A further alternative is that the tumours are of neurogenic origin, similar to that found in the case reported by Neilson (1958). In order to establish this diagnosis, it is necessary to see true nuclear palisading together with evidence of tumour arising from nerve sheaths, as shown in Fig. 5. These features were not seen in any case in this series, and we feel that this possibility can be excluded.

From the clinical point of view, there are no particular features in the adult that are suggestive of a sarcoma. The majority of the tumours occur in the same age group as carcinoma and produce similar symptoms, although a number of the recorded cases of leiomyosarcoma have been in children (Killingsworth, McReynolds, and Harrison, 1953 ; Watson and Anlyan, 1954 ; Merritt and Parker, 1957). Some of the peripheral tumours present a somewhat characteristic appearance on account of their spherical shape and apparent encapsulation, which appears on the radiograph as a coin shadow. Bronchial biopsy may establish the diagnosis in cases where the growth is not peripheral. Williams and Daniel (1950) found suspicious cells on cytological examination of the sputum in their own case, but this method is unlikely to succeed with a welldifferentiated tumour, and it would be difficult for the cytologist to recognize the real nature of the cells in other cases.

The prognosis of pulmonary fibroleiomyosarcoma seems to be related to the degree of differentiation and the frequency of mitotic figures (Stout and Hill, 1958). Patients with well- differentiated tumours amenable to surgery may be expected to do well, and a number of surgical resections have been reported, notably by Watson and Anlyan (1954). Exact diagnosis is important. There is a poor prognosis in the other cases with extensive local spread and distant metastases.

\section{SUMMARY}

Five cases of primary lung fibroleiomyosarcoma are reported. The histological criteria for diagnosis are described and the main pathological and clinical features are discussed. Microscopically, the tumours present a range of appearances from those which are well differentiated to anaplastic tumours with multiple metastases. The prognosis appears to be related to the degree of histological differentiation.

We should like to thank Dr. H. Spencer, Professor H. A. Magnus, and Dr. J. R. Tighe for their advice and criticism. We are very grateful to Dr. S. Robinson, who made case 1 available to us. The photomicrographs were taken by Mr. G. Harwood.

\section{REFERENCES}

Agnos, J. W., and Starkey, G. W. B. (1958). Primary leiomyosarcoma and leiomyoma of the lung. New Engl. J. Med., 258, 12.

Glennie, J. S., Harvey, P., and Jewsbury, P. (1959). Two cases of leiomyosarcoma of the lung. Thorax, 14, 327.

Herovici, C. (1963). A polychrome stain for differentiating precollagen from collagen. Stain Technol., 38, 204.

Killingsworth, W. P., McReynolds, G.S., and Harrison, A. W. (1953). Pulmonary leiomyosarcoma in a child. J. Pediat., 42, 466.

Merritt, J. W., and Parker, K. R. (1957). Intrathoracic leiomyosarcoma. Canad. med. Ass. J., 77, 1031.

Neilson, D. B. (1958). Primary intrapulmonary neurogenic sarcoma. J. Path. Bact., 76, 419.

Pierce, W. F., Alznauer, R. L., and Rolle, C. (1954). Leiomyoma of the lung. Arch. Path., 58, 443.

Stout, A. P., and Hill, W. T. (1958). Leiomyosarcoma of the superficial soft tissues. Cancer (Philad.), 11, 844.

Watson, W. L., and Anlyan, A. J. (1954). Primary leiomyosarcoma of the lung. Ibid., 7,250 .

Williams, R. B., and Daniel, R. A. (1950). Leiomyoma of the lung. J. thorac. Surg., 19, 806.

Yacoubian, H., Connolly, J. E., and Wylie, R. H. (1958). Leiomyosarcoma of the lung. Ann. Surg., 147, 116. 